\title{
Nobelpreis für Robert Wilson und Paul Milgrom: Zwei Ökonomen, die echte Märkte schufen
}

\author{
Robert Wilson und Paul Milgrom erhielten am 10. Dezember 2020 den Preis der \\ Schwedischen Nationalbank für Wirtschaftswissenschaften zu Ehren von Alfred Nobel. \\ Die Ökonomen erhielten den Preis für ihre Beiträge zur Auktionstheorie und für die \\ Entwicklung neuer Auktionsformate.
}

Robert Wilson und Paul Milgrom sind die beiden Ökonomen, die dieses Jahr mit dem Alfred-Nobel-Gedächtnispreis für Wirtschaftswissenschaften ausgezeichnet werden. Die beiden Ökonomen der Stanford University erhalten den Preis für ihre Beiträge zur Auktionstheorie und für die Entwicklung neuer Auktionsformate, so die Königlich Schwedische Akademie der Wissenschaften, welche die Preisträger*innen auswählt. Der Ökonomie-Nobelpreis wurde nicht von Alfred Nobel ins Leben gerufen, sondern als „Preis der Schwedischen Nationalbank in Wirtschaftswissenschaft in Erinnerung an Alfred Nobel" erst 1968 von der schwedischen Zentralbank anlässlich ihres 300-jährigen Bestehens gestiftet und 1969 erstmals verliehen. Gleichwohl haben sich, zum großen Unmut einiger Kritischer, umgangssprachlich die Bezeichnungen als „Nobelpreis für Wirtschaftswissenschaften" oder "Ökonomie-Nobelpreis" durchgesetzt, zumal die Auswahl der Preisträger*innen wie bei den "echten“ Nobelpreisen durch die Königlich Schwedische Akademie der Wissenschaften nach vergleichbaren Kriterien erfolgt. Eine Ausnahme bildet hier allein der Friedensnobelpreis.

In diesem Jahr gab es den Preis also für Fortschritte in der Auktionstheorie und die Entwicklung neuer Auktionsformate. Die Auktionstheorie ist einer der beiden großen Bereiche der sogenannten Theorie des Marktdesigns (Haeringer, 2018), die sich insbesondere mit der Ausgestaltung optimaler Regeln einerseits bei Auktionen und andererseits beim Matching, also der Bildung von Paaren, beschäftigt. Für Auktionen ist charakteristisch, dass mehrere Marktteilnehmende um den Zuschlag für mindestens ein Objekt oder einen Auftrag konkurrieren und der Parameter, der über den Zuschlag entscheidet, ausschließlich der angebotene Preis ist. Auf Matchingmärkten hingegen spielen Preise regelmäBig keine Rolle. Die optimale Zuordnung von Organspenden auf Empfänger*innen oder die möglichst präferenzgerechte

(C) Der/die Autor(en) 2020. Open Access: Dieser Artikel wird unter der Creative Commons Namensnennung 4.0 International Lizenz (https:// creativecommons.org/licenses/by/4.0/deed.de) veröffentlicht.

Open Access wird durch die ZBW - Leibniz-Informationszentrum Wirtschaft gefördert.

* Teile dieses Beitrags erscheinen in einer kürzeren Fassung für pädagogische Zwecke parallel in: Wirtschaftswissenschaftliches Studium.
Verteilung von Schüler*innen auf Schulen erfolgt in aller Regel ohne Rückgriff auf den Preismechanismus. Im Grunde sind Matchingmärkte also eigentlich gerade keine Märkte, zumindest nicht im Sinne von Polanyi $(1944,1957)$, Coase (1937, 1988) oder Williamson (1975), für die der preisgesteuerte Tausch das konstituierende Element eines Marktes ist, das den Markt gerade von anderen Allokationsmechanismen wie Umverteilung, Anordnung oder Reziprozität unterscheidet. Insofern ist der Begriff Marktdesign irreführend, wenn es um Matchingprobleme geht. Der breitere Begriff des Mechanismusdesigns ist hier exakter.

Für ihre Forschung zum optimalen Design von Matchingregeln und zu Bedingungen für stabile Allokationen haben Alvin E. Roth und Lloyd S. Shapley bereits 2012 den Nobelpreis erhalten (Kübler, 2012). Nun ist der Preis erneut für Erkenntnisfortschritte im Bereich des Marktdesigns vergeben worden, aber in diesem Jahr geht es auch wirklich um Märkte. Paul Milgrom und Robert Wilson haben wegweisende Beiträge zur Auktionstheorie geleistet, sodass Märkte und der Preismechanismus als Instrument zur Allokation knapper Ressourcen verwendet werden können und in der Vergangenheit verwendete Allokationsregeln ersetzen können. Das Design optimaler Regeln, damit der Preismechanismus für eine möglichst effiziente Zuteilung von Ressourcen genutzt werden kann, ist allerdings keineswegs so trivial, wie es vordergründig oftmals erscheinen mag.

\section{Auktionstheorie I: Die frühen Jahre}

Dass Preismechanismus nicht gleich Preismechanismus und Markt nicht gleich Markt ist, sondern Marktregeln

Prof. Dr. Justus Haucap ist Direktor des Düsseldorf Institute for Competition Economics (DICE) an der Heinrich-Heine-Universität in Düsseldorf. 
entscheidend für das Marktergebnis sind, ist keine ganz neue Erkenntnis. Traditionell wird zunächst zwischen vier verschiedenen Auktionsformaten unterschieden (etwa Milgrom, 1989, 2004; Klemperer 2004):

- In der Englischen Auktion starten die Angebote bei einem Mindestgebot (das auch null sein kann). Runde für Runde können Bietende dann Gebote abgeben und das jeweils gültige Gebot der Vorrunde erhöhen. Auch kann jeder das gesamte Auktionsgeschehen beobachten, insbesondere wie viel gerade geboten wird. Die Auktion endet, wenn keine höheren Gebote mehr abgegeben werden. Der Bieter mit dem höchsten Gebot erhält den Zuschlag und hat sein Gebot als Preis zu entrichten.

- Die Holländische Auktion wird teilweise auch als Rückwärtsauktion bezeichnet. Bei dieser Auktion startet die Auktion mit einem sehr hohen Preis, der dann nach und nach reduziert wird, bis ein Bieter „zuschlägt“. Für die Durchführung der Auktion wurde traditionell eine rückwärts laufende Auktionsuhr verwendet, die der Bieter dann anhalten kann, wenn er kaufen möchte. Den Zuschlag erhält derjenige, der als erstes die Uhr stoppt. Zu zahlen ist der dann angezeigte Preis.

- Bei der Erstpreisauktion mit verdeckten Geboten (firstprice sealed-bid) geben die Bietenden verdeckt ein einziges Angebot ab, das die anderen nicht einsehen können. Den Zuschlag erhält nach Eingang aller Gebote der Bieter mit dem höchsten Gebot. Zu entrichten ist vom Gewinner der Auktion der Preis, den er geboten hat.

- Im Gegensatz dazu ist bei der Zweitpreisauktion mit verdeckten Geboten (second-price sealed bid) nicht der Höchstpreis, der geboten wurde, zu entrichten, sondern das zweithöchste Gebot. Ansonsten besteht kein Unterschied zur Erstpreisauktion mit verdeckten Geboten.

Die Zweitpreisauktion mit verdeckten Geboten wird auch als Vickrey-Auktion bezeichnet, benannt nach dem Ökonomie-Nobelpreisträger 1996, William Vickrey (Moldovanu, 1996). Vor allem der Beitrag von Vickrey (1961) war für die Auktionstheorie von Bedeutung. Analysiert werden dort Auktionen, bei denen die (risikoneutralen) Bietenden jeweils eine individuelle Wertschätzung (private value) für das Versteigerungsobjekt haben, die nur sie selbst kennen. Vickrey unterstellt, dass diese private Wertschätzung eines jeden Bieters unabhängig ist von der Wertschätzung anderer für das Versteigerungsobjekt. Es gibt für die einzelnen Bietenden in Vickreys Modell keinerlei Unsicherheit über den eigenen, individuellen oder privaten Wert des Objekts - jeder Bietende weiß genau, was inm das Objekt wert ist.
Wie Vickrey (1961) zeigte, sind unter seinen Annahmen die Holländische Auktion und die Erstpreisauktion mit verdeckten Geboten strategisch äquivalent. Das optimale Gebot eines jeden Bieters in der Erstpreisauktion mit verdeckten Geboten entspricht dem Preis, bei dem er in der Holländischen Auktion auch die Uhr stoppen würde. Jedoch gibt es bei diesen beiden Auktionsformaten keine dominanten Strategien für die Bietenden, weil diese von den Geboten der anderen abhängen. Durch ein geringeres Gebot spart man einerseits Geld, riskiert aber andererseits, den Zuschlag gar nicht zu erhalten. Optimale Gebote müssen hier eine Balance finden.

Strategisch äquivalent sind zudem auch die Englische Auktion und die Zweitpreisauktion mit verdeckten Geboten. In der Englischen Auktion steigt jeder Bieter optimalerweise dann aus, wenn das gültige Gebot die eigene Wertschätzung für das Objekt übersteigt. Durch einen früheren Ausstieg aus der Auktion kann der Bieter nichts gewinnen. Ebenso bringt es in der Zweitpreisauktion mit verdeckten Geboten dem Bieter keinen Vorteil, ein Gebot abzugeben, das unter der eigenen Wertschätzung liegt, da im Erfolgsfall ja ohnehin nur das zweithöchste Gebot zu zahlen ist. Optimal ist es daher, seine eigene Wertschätzung als Gebot zu wählen. Im Gegensatz zur Holländischen Auktion und zur Erstpreisauktion mit verdeckten Geboten haben somit die Englische Auktion und die Zweitpreisauktion mit verdeckten Geboten dominante Strategien.

Die von Vickrey (1961) getroffene Annahme, dass die Wertvorstellungen der Bietenden individuell verschieden und voneinander völlig unabhängig sind (private values), dürfte vielleicht für Privatleute, die Objekte für den eigenen $\mathrm{Ge}$ brauch ersteigern, zutreffend sein. Bei Schürfrechten oder Frequenznutzungsrechten, die von Unternehmen ersteigert werden, um damit auf einem nachgelagerten Markt Geld zu verdienen, dürfte diese Annahme jedoch selten zutreffen. In der Regel sind die mit Hilfe dieser Rechte erzielbaren Erlöse für alle Bietenden unsicher. Auch bei Objekten, bei denen der spätere Wiederverkaufswert eine Rolle spielt, dürfte die Annahme schwer zu halten sein, dass die Einschätzungen der Bietenden über den Wert des Auktionsobjekts voneinander unabhängig sind. Vielmehr dürften die Bewertungen sich gegenseitig beeinflussen.

Robert B. Wilson $(1969,1977)$ war es nun, der die Auktionstheorie zunächst entscheidend weiterentwickelte, indem er Fälle betrachtete, in denen die Wertschätzung der Bietenden für ein Objekt eben nicht voneinander unabhängig ist. Das typische Beispiel hier sind Förderrechte für Ölfelder, bei denen die Bietenden nicht genau wissen, wie groß bzw. ergiebig ein Ölfeld ist. Wäre die Menge an förderbarem ÖI von vornherein exakt bekannt und würden sich die Ölfirmen nicht anderweitig unterscheiden, sollte das Förderrecht prinzipiell jedem Ölkonzern gleich viel wert sein. Weiß 
aber niemand genau, wie viel Öl auf einem Ölfeld gefördert werden kann, so können sich darüber unterschiedliche Einschätzungen bei den Bietenden entwickeln. Diese unterschiedlichen Einschätzungen können sich etwa ergeben, wenn Probebohrungen zu unterschiedlichen Ergebnissen kommen oder - ökonomisch ausgedrückt - die Bietenden unterschiedliche Signale erhalten. In diesem Fall ist leicht nachzuvollziehen, dass die Bietenden etwas aus dem Bietverhalten ihrer Konkurrenz lernen könnten, sofern dies beobachtet werden kann, wie etwa in der Englischen Auktion. Der Wert des Ölförderrechts ist somit prinzipiell für alle Ölkonzerne identisch (common value), aber weil sich die Erwartungen aufgrund verschiedener Signale unterscheiden, sind die Einschätzungen der Bietenden über den „wahren“ Wert nicht mehr unabhängig voneinander, sofern sie direkt oder indirekt in Erfahrung bringen können, von welchen Erwartungen die anderen Bietenden ausgehen.

Wilson (1969) hat nun gezeigt, dass - insbesondere (aber nicht nur) bei der Holländischen Auktion und Auktionen mit verdeckten Geboten, bei denen die Bietenden nichts aus dem Verhalten anderer lernen können - der sogenannte Fluch des Gewinnens (winner's curse) auftritt. Sofern sich Bietende nicht in ihren Risikoeinstellungen unterscheiden, wird derjenige das höchste Gebot abgeben oder die Auktionsuhr als erstes stoppen und so den Zuschlag in der Auktion erhalten, der den tatsächlichen Wert des Objekts - also etwa des Ölförderrechts - am meisten überschätzt. Die Botschaft, eine Auktion gewonnen zu haben, dürfte in diesem Fall eine schlechte Nachricht sein. Rationale Bietende werden daher vorsichtshalber etwas niedriger bieten, um zu vermeiden, dass sich das Gebot im Nachhinein als zu hoch erweist. Die optimale Bietstrategie ist in einer solchen Situation deutlich schwieriger zu ermitteln.

Milgrom (1981a, 1981b) sowie Milgrom und Weber (1982) haben die Theorie Wilsons entscheidend weiterentwickelt, indem sie Auktionen analysierten, bei denen die Bietenden weder eine völlig unabhängige, individuelle Wertschätzung (private value) noch eine vollkommen voneinander abhängige, gemeinsame Wertschätzung (common value) für ein Objekt haben, sondern beides eine Rolle spielt. Wie Milgrom und Weber (1982) zeigen, lässt in einem solchen Kontext die Englische Auktion tendenziell (etwas) höhere Erlöse erwarten als die Zweitpreisauktion mit verdeckten Geboten, sofern die privaten Signale, welche die Bietenden erhalten, miteinander verbunden (affiliated) sind. Verbundenheit ist dabei eine Form der positiven Korrelation. Während Bietende bei der Zweitpreisauktion mit verdeckten Geboten nicht aus den Geboten der anderen lernen können, ergeben sich bei der Englischen Auktion Lerneffekte, da die Bietenden ihre Gebote gegenseitig beobachten und daraus Rückschlüsse auf die jeweiligen privaten Signale der anderen ziehen können. Die Zweitpreisauktion mit verdeckten Geboten wie- derum führt tendenziell zu höheren erwarteten Erlösen als die Holländische Auktion und die Erstpreisauktion mit verdeckten Geboten. Dass in diesem Szenario, das die Realität oftmals am besten widerspiegeln dürfte, die Englische Auktion die höchsten Erlöse erwarten lässt, mag die weite Verbreitung von Englischen Auktionen als Auktionsform, etwa bei E-Bay, bei Auktionen von Fundsachen, Kunstauktionen oder der Zwangsversteigerung von Immobilien, mit erklären. Auch der Fluch des Gewinnens ist in der Englischen Auktion weniger ausgeprägt, da durch die Beobachtung der anderen Gebote Informationen preisgegeben werden, was wiederum die Bietenden zu aggressiveren Bietstrategien veranlasst und letztlich auch zu höheren Auktionserlösen führt. Milgrom und Weber (1982) zeigen in ihrem Beitrag auch, dass Verkäufer*inne in einer Auktion höhere Erlöse erzielen können, wenn sie ihre eigenen Informationen, etwa in Form eigener Expertisen, für die Kaufinteressenten offenlegen.

Die theoretischen Vorhersagen der Modelle von Wilson und Milgrom sind in zahlreichen empirischen und experimentellen Untersuchungen inzwischen überprüft und belegt worden. Das Hintergrundpapier der Königlich Schwedischen Akademie der Wissenschaften (2020) nennt hier zahlreiche Quellen. Allgemeine Überblicke über die experimentelle Forschung zur Auktionstheorie finden sich bei Kagel (1995) sowie Kagel und Levin (2014).

\section{Frequenzauktionen als Beschleuniger der Forschung}

War die Auktionstheorie lange etwas für ausgemachte Fachleute, erfuhr die Forschung zu Auktionen einen ungeheuren Schub durch die Entscheidung des US-Kongresses 1993, der US-amerikanischen Regulierungsbehörde für den Telekommunikationssektor, der Federal Communications Commission (FCC), die Möglichkeit einzuräumen, Rechte zur Nutzung des Frequenzspektrums per Auktion zu versteigern. Zuvor waren die Rechte - genau wie in Deutschland und eigentlich allen anderen Ländern - entweder administrativ anhand eines festgelegten Kriterienkatalogs an Bewerber*innen vergeben worden - ein Verfahren, das auch als „Schönheitswettbewerb“ bezeichnet wird - oder aber durch sogenannte Spektrumslotterien. In den USA wurden seit 1982 Teile des Frequenzspektrums per Los vergeben, weil die administrativen „Schönheitswettbewerbe" der FCC zu viel Zeit in Anspruch nahmen und so viele Teile des Frequenzspektrums lange ungenutzt brach lagen. Die vom US-Kongress daraufhin 1982 eingeführten Spektrumslotterien waren in dem Sinne erfolgreich, dass die Frequenznutzungsrechte schneller vergeben wurden. Allerdings führten die Lotterien auch dazu, dass sich alle möglichen Interessent ${ }^{*}$ innen um Spektrumsrechte bewarben, um diese Rechte dann typischerweise für viel Geld weiterzuverkaufen (McMillan, 1994). Damit wurde deutlich, auf welche Einnahmen der Staat durch die unentgeltliche 
Vergabe verzichtete. Manche Lotterien zogen mehrere 100.000 Bewerbungen an, in der Hoffnung, die Rechte dann profitabel weiter verkaufen zu können.

Nach der Entscheidung des US-Kongresses 1993 sollte der Markt bzw. der Preismechanismus genutzt werden, um herauszufinden, welcher Bewerber die höchste Wertschätzung für die verschiedenen Teile des Frequenzspektrums bzw. die entsprechenden Nutzungsrechte hat. Grund für den Stimmungswechsel im US-Kongress war allerdings nicht die Überzeugung, dass Auktionen eine effiziente Allokation gewährleisten, sondern vor allem die Aussicht auf zusätzliche Staatseinnahmen (Herzel, 1998).

Ronald Coase, Ökonomie-Nobelpreisträger 1991 (Cortekar und Groth, 2010), hatte schon 1959 vorgeschlagen, Auktionen zur Allokation des Frequenzspektrums zu nutzen (Coase, 1959), wobei die Idee ursprünglich auf Herzel (1951) zurückzugehen scheint, wie Coase selbst in seinem Aufsatz betont hat. Umgesetzt wurde die Idee allerdings nicht zuerst in den USA, sondern in Neuseeland, wo schon 1990 Rechte am Frequenzspektrum für Radio, Fernsehen und Mobiltelefonie versteigert wurden. Auf Rat des Beratungshauses NERA führte die neuseeländische Regierung Zweitpreisauktionen mit verdeckten Geboten durch (McMillan, 1994, 148 ff.). Dies führte jedoch dazu, dass die Gewinner*innen der Auktion Preise zahlten, die weit unter ihren Geboten lagen. In einem Extremfall hatte ein Unternehmen 100.000 NZ\$ geboten, musste aber nur das zweithöchste Gebot in Höhe von 6 NZ\$ zahlen. In einem anderen Fall lag das Höchstgebot bei 7 Mio. NZ\$, das zweite jedoch nur bei 5.000 NZ\$. Die erwarteten Einnahmen blieben insgesamt sehr deutlich unter den Erwartungen: Die Mobilfunklizenzen brachten dem neuseeländischen Staat 36 Mio. NZ\$ statt der erwarteten 240 Mio. NZ\$, die NERA prognostiziert hatte. In der öffentlichen Darstellung war die Auktion für die neuseeländische Regierung daher kein Erfolg.

In den USA wurden die Schwachpunkte des neuseeländischen Auktionsdesigns vermieden und zahlreiche Auktionstheoretiker*innen herangezogen, um das Design für die Auktionen von Frequenzspektren zu entwickeln, darunter auch Paul Milgrom und Robert Wilson. Sehr lesenswert ist in diesem Kontext der Bericht von Kwerel und Rosston (2000), den beiden Ökonomen, welche die Idee der Versteigerung des Frequenzspektrums innerhalb der FCC maßgeblich vorangetrieben haben.

Im Gegensatz zu den simplen Auktionen, bei denen einzelne Objekte meistbietend versteigert werden und das Ziel allein darin besteht, möglichst hohe Erlöse zu generieren, sind bei Frequenzauktionen zahlreiche Faktoren zu berücksichtigen, die das optimale Auktionsdesign komplex machen (Cramton, 2015). In den USA etwa waren - anders als in Deutschland, Frankreich oder anderen Ländern in Europa - einige Bewerber*innen nur am Frequenzspektrum in bestimmten Landesteilen und in bestimmten USBundesstaaten interessiert, nicht aber an einer US-weiten Abdeckung. Zugleich war davon auszugehen, dass ein Aufbau von Mobilfunknetzen in aneinandergrenzenden Regionen sowohl aus Kostensicht als auch aus Marketinggesichtspunkten günstiger war als der Aufbau von Mobilfunknetzen in völlig separaten Bundesstaaten. Aufgrund der Verbund- und Größenvorteile bestand also zwischen den Rechten zur Frequenznutzung in aneinandergrenzenden Gebieten ein komplementäres Verhältnis. Des Weiteren verfolgten verschiedene Bietende unterschiedliche Geschäftsmodelle und beabsichtigen, unterschiedliche Technologien einzusetzen, die eine unterschiedliche Organisation des Spektrums erfordern (etwa Cramton, 2013). Und schließlich begrenzen Interferenzen benachbarter Frequenzbänder die Substituierbarkeit einzelner Teile des Frequenzspektrums. Die simple Verwendung einer der vier genannten Auktions-Grundformate, wie etwa die in Neuseeland zuvor verwendete Zweitpreisauktion mit verdeckten Geboten, erschien daher nicht ratsam.

\section{Auktionstheorie II: Neue Auktionsformate}

Milgrom und Wilson entwickelten gemeinsam mit Preston McAfee nun zunächst die sogenannte simultane Mehrrundenauktion (SMRA), eine Weiterentwicklung der Englischen Auktion (Kwerel und Rosston, 2000). Versteigert werden bei der SMRA mehrere Objekte, deren Wert nicht unabhängig voneinander ist, wie regionale Frequenznutzungsrechte. Alle Rechte werden simultan versteigert und nicht etwa nacheinander, um so das Problem zu umgehen, dass Bietende bei einer sequenziellen Auktion beim Gebot auf ein Recht noch nicht wissen, ob sie für später zu versteigernde Rechte auch die Höchstbietenden sein werden. Es werden somit parallel möglichst viele Rechte versteigert, um Komplementaritäten ausschöpfen zu können. Alle Frequenzrechte starten simultan mit einem Mindestpreis. Die Bietenden können dann Runde für Runde ihr Gebot für jedes Frequenzrecht erhöhen. Die Auktion wird so lange fortgesetzt, bis kein Bieter mehr ein höheres Gebot für ein Frequenzrecht abgibt. Zu diesem Zeitpunkt werden für alle Frequenzrechte die Zuschläge an den Meistbietenden erteilt.

Bei der Auktion wurde zudem eine sogenannte Aktivitätsregel eingeführt, die Bietende daran hindert, sich, wie dies etwa bei E-Bay möglich ist, bis zum Ende der Auktion gar nicht mitzubieten und dann erst am Ende „zuzuschlagen“. Stattdessen muss der Bieter in jeder Runde eine Mindestaktivität entfalten, um auch weiter bieten zu dürfen. Mindestaktivität bedeutet, dass man entweder ein neues Gebot abgeben muss oder selbst in der Vorrunde der Höchstbietende für ein bestimmtes Recht war. Zudem müssen Bietende vor Auktionsbeginn eine Kaution einreichen, 
deren Höhe bestimmt, auf wie viel Spektrum der Bieter bieten darf. Die Mindestaktivitätsregel startet in der Regel bei $80 \%$ der durch die Kaution determinierten Menge und steigt dann stufenweise auf $100 \%$. Wenn ein Bieter in einer Runde unter dem geforderten Prozentsatz liegt, wird die Berechtigung zu bieten in der folgenden Runde proportional reduziert. So wird gewährleistet, dass die Auktion relativ zügig verläuft. Bei der erstmaligen Nutzung der SMRA als Auktionsformat für nur zehn Lizenzen 1994 wurden bereits 617 Mio. US-\$ eingenommen. Aufgrund dieses Erfolgs für die FCC folgten zahlreiche Auktionen dieses Formats in den USA, Deutschland und anderen Ländern (Milgrom, 2004; Bichler und Goeree, 2017; Goetzendorff et al., 2018).

Obwohl die Auktionen besser funktionierten als in Neuseeland, hatten die ersten Frequenzauktionen noch immer gewisse Mankos: Bei den ersten Auktionen wurden sämtliche Gebote und die Identität der Bietenden nach jeder Runde offengelegt und somit vollständige Transparenz geschaffen. Diese Regel ermöglichte es den Bietenden jedoch, z. B. mit den Ziffern ihrer Gebote Signale zu senden und so kollusives Verhalten zu induzieren (Cramton und Schwartz, 2002). Heute sind die Auktionen daher in aller Regel in dem Sinne anonym, dass nur die Höchstgebote bekanntgegeben werden und dies auch, ohne die Identität der Bietenden preiszugeben. Zudem dürfen Gebote nur in zuvor festgelegten Schritten - z.B. genau $5 \%$ - erhöht werden, um kollusives Verhalten zu vermeiden.

Eine später eingeführte Vereinfachung der SMRA bestand zudem in der Nutzung sogenannter Taktauktionen (clock auctions) für abstrakte Frequenznutzungsrechte. Die Bietenden geben dann nicht Gebote für konkrete Blöcke des Spektrums ab, sondern bieten abstrakt auf eine Menge an Spektrum. So kann das Frequenzband am Ende der Auktion technisch effizient zugeteilt werden, sodass die Bietenden zusammenhängende Teile des Spektrums erhalten. Versteigert eine Regulierungsbehörde etwa 50 $\mathrm{MHz}$ eines Frequenzbandes in gepaarten $5+5 \mathrm{MHz}-$ Paketen, so gibt es fünf mögliche Pakete, die prinzipiell ziemlich gut, wenn auch aufgrund der unterschiedlichen Lage im Frequenzband nicht unbedingt perfekt substituierbar sind. Bei der Taktauktion nennt der Auktionator oder die Auktionatorin dann einen Preis und fragt die Bietenden, wie viele Pakete sie zu diesem Preis ersteigern möchten. Besteht ein Nachfrageüberhang, erhöht der Auktionator den Preis so lange, bis Angebot und Nachfrage übereinstimmen. Dies bestimmt die Zahl der Pakete, die jeder Bietende gewonnen hat, und ihren Preis.

Diese Methode vereinfacht das Bieten und verhindert so strategisches, wettbewerbswidriges Verhalten. Zudem garantiert die Methode, dass alle Pakete zum gleichen Preis verkauft werden und jeder Bietende ein zusammenhän- gendes Spektrum gewinnt, sodass technische Ineffizienzen vermieden werden. Ein Defizit der Auktionen bestand jedoch noch immer darin, dass Bietende nicht explizit auf Lizenzpakete bieten konnten. Das heißt, die bisher genannten Auktionsformate leiden darunter, dass Komplementaritäten noch nicht ideal ausgeschöpft werden, weil kein gemeinsames Gebot auf zwei oder mehr Objekte möglich ist, sondern für jedes vordefinierte Paket getrennt geboten wird. So kann es vorkommen, dass ein Bieter den Zuschlag für ein Paket erhält, obwohl er es eigentlich nur in Kombination mit einem anderen Teil des Spektrums wertschätzt.

Ausubel und Milgrom (2002a, 2002b) haben daher die „kombinatorische Taktauktion“ (combinatorial clock auction) entwickelt (auch Cramton, Shoham und Steinberg, 2006). In der ersten Stufe der Auktion können Bietende als Reaktion auf die im Takt steigenden Preise ihre Gebote bestätigen, bis für jedes Objekt nur noch ein Bieter verbleibt, d. h. es erfolgt eine normale Taktauktion. Anschließend folgt jedoch als eine zweite Auktionsstufe eine Zweitpreisauktion mit verdeckten Geboten, bei der die Bietenden verdeckt verbesserte Gebote für Kombinationen von Objekten abgeben können. Die Allokation der Auktionsobjekte erfolgt dann so, dass die in den Geboten reflektierte Wertschätzung für die Objekte maximiert wird, um eine effiziente Allokation zu erreichen. Auch wenn die kombinatorische Taktauktion Probleme mit sich bringen kann, wie das strategische Hochbieten für Objekte, um die Konkurrenz zu höheren Zahlungen zu zwingen (Bichler und Goeree, 2017), wurde die Auktion 2008 erstmals erfolgreich in Großbritannien zur Versteigerung von Rechten am Frequenzspektrum angewendet und später in vielen anderen europäischen Staaten. Der große Vorteil dieser Auktionsform ist die Möglichkeit, auf individuell gebildete Pakete zu bieten, sodass Komplementaritäten besser berücksichtigt werden (Cramton, 2013; Ausubel und Baranov, 2014).

Die jüngste Entwicklung besteht nun im Design von „Anreizauktionen“, bei denen es eigentlich um eine Kombination von zwei Auktionen geht (Wilson, 1985). Die FCC hat dieses Format 2017 eingesetzt, um Frequenznutzungsrechte neu zu verteilen. Auf der ersten Stufe wurde in einer „Beschaffungsauktion" ein Preis bestimmt, zu dem Rundfunksender freiwillig bestehende Frequenznutzungsrechte aufgeben. In der zweiten Auktion wurde das frei gewordene Spektrum neu versteigert. 2017 gaben bei der „Beschaffungsauktion“ 14 Rundfunksender in den USA Frequenznutzungsrechte auf und erhielten dafür 10,1 Mrd. US-\$. Bei der anschließenden Auktion auf der zweiten Stufe wurden Rechte an $70 \mathrm{MHz}$ Frequenzspektrum für 19,8 Mrd. US-\$ versteigert und zudem $14 \mathrm{MHz}$ überschüssiges Spektrum einbehalten. Die zwei Stufen der Anreizauktion brachten den US-Steuerzahler*innen somit knapp 10 Mrd. US-\$ ein und setzten große Teile des Spektrums für zukünftige Nutzungen frei. Die Auktion darf damit wohl als Erfolg bezeichnet werden (Milgrom und Segal, 2017, 2020). 


\section{Auktionen in Energie- und Entsorgungswirtschaft}

Neben den Auktionen von Rechten am Frequenzspektrum hat sich Robert Wilson auch mit Auktionen beschäftigt, in denen homogene Anteile eines bestimmten Gutes versteigert werden (share auctions). Beispiele sind Staatsschuldtitel oder an der Strombörse gehandelte Bezugsrechte für Elektrizität. Wilson (1979) entwickelte zunächst ein Modell, in dem er sowohl Einheitspreisauktionen betrachtete, bei denen für jeden Anteil letztlich derselbe (markträumende) Preis gezahlt wird, als auch diskriminierende Auktionen, bei denen für jeden Anteil der angegebene Gebotspreis zu zahlen ist (pay as bid). Eine wesentliche Einsicht von Wilson (1979) bestand darin, dass Bietende in ihrer Strategie berücksichtigen werden, wie das Gebot für jeden weiteren Anteil den Preis für die anderen Anteile beeinflusst, d.h. wie das Gebot auf die letzte (marginale) Einheit den Preis für die inframarginalen Einheiten verändert. Im Vergleich zur Versteigerung einzelner Objekte haben Bietende einen Anreiz, zurückhaltender und weniger aggressiv zu bieten. Das Problem der strategischen Kapazitätszurückhaltung ist etwa im Stromgroßhandel virulent. In solchen Auktionen können sich Teilnehmende explizit absprechen oder indirekt koordinieren, um den Preis zu beeinflussen und den Wettbewerb zu schwächen oder auszuschalten. Für das Design von Strombörsen auf liberalisierten Elektrizitätsmärkten wurde dieses Modell von Wilson $(2002,2008)$ und anderen weiterentwickelt, wie Klemperer und Meyer (1989), Joskow und Tirole (2000), Kremer und Nyborg (2004), Cramton und Ockenfels (2012).

Auch in anderen Bereichen erfreuen sich Auktionen einer wachsenden Beliebtheit. So wird etwa bei der Subventionsvergabe zur Errichtung von Erzeugungskapazitäten für Strom aus erneuerbaren Energien nach EEG sowie KraftWärme-Kopplung (KWK) verstärkt auf wettbewerbliche Ausschreibungen gesetzt (Bundesnetzagentur, 2020). Im stark öffentlich bezuschussten Regionalverkehr der Bahn werden vermehrt Ausschreibungen genutzt. Beim beschlossenen Kohleausstieg können Stromproduzierende in acht sequenziellen Auktionen um die Stillegung ihrer Steinkohlekraftwerke bieten. Wer die geringste Entschädigung pro Tonne $\mathrm{CO}_{2}$-Emission fordert, bekommt den Zuschlag für die Stilllegung seines Steinkohlekraftwerks. $\mathrm{Ob}$ das Auktionsdesign und die Zuschlagsregel wirklich optimal sind, sei allerdings dahingestellt. Effiziente Kraftwerke, die relativ wenig $\mathrm{CO}_{2}$ pro MWh Strom ausstoßen, können relativ hohe Gebote in Euro pro stillgelegter MW Kraftwerkskapazität abgeben und dennoch den Zuschlag erhalten, sodass sich die frühe Stilllegung effizienter Kraftwerke gegebenenfalls besonders lohnen könnte. Vielleicht hätte man beim Entwurf des Kohleausstiegsgesetzes und dem Design der Ausstiegsauktionen auch Auktionstheoretiker*innen stärker um Rat fragen sollen.
Es gibt weitere Bereiche, in denen zwar Ausschreibungen und Auktionen genutzt werden, das Format der Ausschreibung oder Auktion aber nicht optimal ist. Bei der Müllentsorgung etwa zeigt sich, dass es erhebliche Kostenvorteile gibt, wenn ein Unternehmen den Zuschlag in benachbarten Gebieten erhält (Böckers et al, 2016). Gleichwohl werden die Aufträge zur Restmüllerfassung - sicher auch aufgrund der Zuständigkeit unterschiedlicher Kommunen - in der Regel nicht simultan ausgeschrieben, obwohl sich gerade hier aufgrund der regionalen Skalenvorteile die von Milgrom und Wilson entwickelte simultane Mehrrundenauktion oder die kombinatorische Taktauktion anbieten würde. Weil aber die Ausschreibungen heute in den Kommunen zeitversetzt jeweils nur für die eigene Kommune erfolgen, erhalten die Kommunen oft nur eine beschränkte Zahl von Angeboten von den Entsorgungsbetrieben, die bereits in einer Nachbarkommune tätig sind. Durch eine simultane Vergabe mit der Möglichkeit auch in Kombination auf Pakete zu bieten, ließe sich der Wettbewerb in der Müllentsorgung steigern.

\section{Schlussbemerkungen}

Die Auktionstheorie ist ein sehr mathematisches Feld der Ökonomie und für Außenstehende nicht immer leicht zu durchdringen. Gleichwohl ist ihr praktischer Nutzen außerordentlich. Wenige Ökonom*innen dürften so viel für das Entstehen echter Märkte geleistet haben wie Robert Wilson und Paul Milgrom mit dem Design verschiedener Auktionen, die ermöglicht haben, dass andere Allokationsformen durch den Preismechanismus abgelöst wurden. Im Sinne von Karl Polanyi (1944) haben Milgrom und Wilson also selbst zumindest eine kleine Transformation bewirkt, bei der „Schönheitswettbewerbe“ und Lotterien durch Märkte abgelöst wurden. Im Grunde greift die jetzt prämierte Forschung von Milgrom und Wilson eine alte Einsicht von Ronald Coase $(1988,8)$ auf, dass Märkte - anders als oft in der Ökonomie angenommen - nicht einfach da sind, sondern Märkte oftmals erst geschaffen werden, etwa durch die Schaffung von Verfügungsrechten und auch durch das Design von Marktregeln (ausführlich Haucap, 2017). Von deren Ausgestaltung hängt $a b$, ob eine preisgesteuerte Allokation, also Märkte, effiziente Ergebnisse hervorbringen oder nicht. Es ist sicher auch kein Zufall, dass Paul Milgrom sich in vielen seiner Beiträge mit der Frage beschäftigt hat, wie und wann genau Märkte entstehen. Zu seinen fünf meistzitierten Beiträgen gehört der Aufsatz von Milgrom, North und Weingast (1990) über die Institutionen, welche die Entstehung mittelalterlicher Märkte in der Champagne ermöglicht haben. Schließlich sei angemerkt, dass gerade Paul Milgrom auch in vielen anderen Bereichen der Ökonomik Spuren hinterlassen hat. Prominent sind sicher sein mit John Roberts verfasstes Lehrbuch zur Organisationsökonomik (Milgrom und Roberts, 1992), seine Beiträge zur Theorie der Unternehmung (etwa Milgrom und Roberts, 1995), zur Erklärung von Verdrängungspreisen (Milgrom und Roberts, 1982) 
sowie zur mathematischen Wirtschaftstheorie (etwa Milgrom und Segal, 2002), um nur einige zu nennen.

\section{Literatur}

Ausubel, L. und O. Baranov (2014), Market Design and the Evolution of the Combinatorial Clock Auction, American Economic Review, 104, 446-451.

Ausubel, L. M. und P. Milgrom (2002a), Ascending Auctions with Package Bidding, The B. E. Journal of Theoretical Economics, 1, 1-44.

Ausubel, L. M. und P. Milgrom (2002b), Package Bidding: Vickrey vs Ascending Auctions, Revue Economique, 3, 391-402.

Bichler, M. und J. Goeree (Hrsg.) (2017), Handbook of Spectrum Auction Design, Cambridge University Press.

Böckers, V., L. Hardorp, J. Haucap, U. Heimeshoff, N. Gösser und S. Thorwarth (2016), Wettbewerb in der Restmüllerfassung: Eine empirische Analyse der Anbieterstruktur, List-Forum für Wirtschafts- und Finanzpolitik, 42, 423-440.

Coase, R. H. (1937), The Nature of the Firm, Economica, 4, 386-405.

Coase, R. (1959), The Federal Communications Commission, Journal of Law and Economics, 2, 1-40.

Coase, R. H. (1988), The Firm, the Market, and the Law, University of Chicago Press.

Cortekar, J. und M. Groth (2010), Die Bedeutung von Transaktionskosten und Verfügungsrechten: Eine Würdigung zum 100. Geburtstag von Ronald H. Coase, Wirtschaftsdienst, 90, 826-830.

Cramton, P. (2013), Spectrum Auction Design, Review of Industrial Organization, 42, 161-190.

Cramton, P. (2015), Market Design in Energy and Communications, Working paper, http://www.cramton.umd.edu/papers2015-2019/cramton-market-design-in-energy-and-communications.pdf (4. Dezember 2020).

Cramton, P. und O. Ockenfels (2012), Economics and Design of Capacity Markets for the Power Sector, Zeitschrift für Energiewirtschaft, 36, 113-134.

Cramton, P. und J. Schwartz (2002), Collusive Bidding in the FCC Spectrum Auctions, Contributions to Economic Analysis \& Policy, 1, 1-17.

Cramton, P., Y. Shoham und R. Steinberg (2006), Combinatorial Auctions, MIT Press.

Bundesnetzagentur (2020), Ausschreibungen für EE- und KWK-Anlagen, https://www.bundesnetzagentur.de/DE/Sachgebiete/ElektrizitaetundGas/Unternehmen_Institutionen/Ausschreibungen/Ausschreibungen_ node.html ( 8. Dezember 2020).

Goetzendorff, A., M. Bichler und J. K. Goeree (2018), Synergistic Valuations and Efficiency in Spectrum Auctions, Telecommunications Policy, 42, 91-105.

Haeringer, G. (2018), Market Design: Auctions and Matching, MIT-Press.

Haucap, J. (2017), The Rule of Law and the Emergence of Market Exchange: A New Institutional Economic Perspective, in U. von Alemann, F. Briesen und L. Q. Khanh (Hrsg.), The State of Law: Comparative Perspectives on the Rule of Law, Düsseldorf University Press, 143-172.

Herzel, L. (1951), 'Public Interest' and the Market in Color Television Regulation, University of Chicago Law Review, 18, 802-816.

Herzel, L. (1998), My 1951 Color Television Article, Journal of Law and Economics, 41, 523-528.

Joskow, P. L. und J. Tirole (2000), Transmission Rights and Market Power on Electric Power Networks, RAND Journal of Economics, 31, 450-487.

Kagel, J. H. (1995), Auctions: A Survey of Experimental Research, in J. H. Kagel und A. E. Roth (Hrsg)., Handbook of Experimental Economics, Bd. 2, Cambridge University Press.

Kagel, J. H. und D. Levin (2014), Auctions: A Survey of Experimental Research. Klemperer, P. (2004), Auctions: Theory and Practice, Princeton University Press.
Klemperer, P. D. und M. A. Meyer (1989), Supply Function Equilibria in Oligopoly under Uncertainty, Econometrica, 57, 1243-1277.

Königlich Schwedische Akademie der Wissenschaften (2020), Scientific Background Paper in the Sveriges Riksbank Prize in Economic Sciences in Memory of Alfred Nobel 2020.

Kremer, I. und K. G. Nyborg (2004), Divisible-Good Auctions: The Role of Allocation Rules, RAND Journal of Economics, 35, 147-159.

Kübler, D. (2012), Stabile Allokationen und Matching-Märkte: Zum Nobelpreis für Wirtschaftswissenschaften an Lloyd S. Shapley und Alvin E. Roth, Wirtschaftsdienst, 92, 843-847.

Kwerel, E. und G. Rosston (2000), An Insiders' View of FCC Spectrum Auctions, Journal of Regulatory Economics, 17, 253-289.

McMillan, J. (1994), Selling Spectrum Rights, Journal of Economic Perspectives, 8(3), 145-162.

Milgrom, P. (1981a), Good News and Bad News: Representation Theorems and Applications, Bell Journal of Economics, 12, 380-391.

Milgrom, P. (1981b), Rational Expectations, Information Acquisition, and Competitive Bidding, Econometrica, 49, 921-944.

Milgrom, P. (1989), Auctions and Bidding: A Primer, Journal of Economic Perspectives, 3(3), 3-22.

Milgrom, P. (2004), Putting Auction Theory to Work, Cambridge University Press.

Milgrom, P., D. C. North und B. R. Weingast (1990), The Role of Institutions in the Revival of Trade: The Law Merchant, Private Judges, and the Champagne Fairs, Economics and Politics, 2, 1-23.

Milgrom, P. und J. Roberts (1982), Predation, Reputation, and Entry Deterrence, Journal of Economic Theory, 27, 280-312.

Milgrom, P. und J. Roberts (1992), Economics, Organization \& Management, Prentice Hall.

Milgrom, P. und J. Roberts (1995), Complementarities and Fit Strategy, Structure, and Organizational Change in Manufacturing, Journal of ACcounting and Economics, 19, 179-208.

Milgrom, P. und I. Segal (2002), Envelope Theorems for Arbitrary Choice Sets, Econometrica, 70, 583-601.

Milgrom, P. und I. Segal (2017), Designing the US Incentive Auction, in M. Bichler und J. Goeree (Hrsg.), Handbook of Spectrum Auction Design, Cambridge University Press, 803-812.

Milgrom, P. und I. Segal (2020), Clock Auctions and Radio Spectrum Reallocation, Journal of Political Economy, 128, 1-31.

Milgrom, P. und R. J. Weber (1982), A Theory of Auctions and Competitive Bidding, Econometrica, 50, 1089-1122.

Moldovanu, B. (1996), William Vickrey und die Auktionstheorie: Anmerkungen zum Nobelpreis 1996, Wirtschaftsdienst, 76, 651-656.

Polanyi, K. (1944), The Great Transformation, New York.

Polanyi, K. (1957), The Economy as Instituted Process, in K. Polanyi, C. M. Arensberg und H. W. Pearson (Hrsg.), Trade and Market in the Early Empires, Glencoe, 243-270.

Vickrey, W. (1961), Counterspeculation, Auctions, and Competitive SealedTenders, Journal of Finance, 16, 8-37.

Williamson, O. E. (1975), Markets and Hierarchies.

Wilson, R. B. (1969), Competitive Bidding with Disparate Information, Management Science, 15, 446-448.

Wilson, R. B. (1977), A Bidding Model of Perfect Competition, Review of Economic Studies, 44, 511-518.

Wilson, R. B. (1979), Auctions of Shares, Quarterly Journal of Economics, 93, 675-689.

Wilson, R. B. (1985), Incentive Efficiency of Double Auctions, Econometrica, $53,1101-1115$

Wilson, R. B. (2002), Architecture of Power Markets, Econometrica, 70, $1299-1340$.

Wilson, R. B. (2008), Supply Function Equilibrium in a Constrained Transmission System, Operations Research, 56, 369-382.

Title: Nobel Prize for Robert Wilson and Paul Milgrom: Two Economists who Created Real Markets

Abstract: This article summarises the research of the 2020 Nobel laureates in economic sciences, Robert Wilson and Paul Milgrom, who received the prize for improvements to auction theory and inventions of new auction formats. The two economists have not only advanced auction theory, but also used their results to developed new auction formats that have globally substituted other means of resource allocation to the benefit of buyers, sellers and taxpayers around the globe.

JEL Classification: D44, D47, L96, A11 